\title{
Deterministic and Stochastic Study of Wind Farm Harmonic Currents
}

\author{
Luis Sainz, Juan Jose Mesas, Remus Teodorescu, Senior Member, IEEE, and Pedro Rodriguez, Member, IEEE
}

\begin{abstract}
Wind farm harmonic emissions are a well-known power quality problem, but little data based on actual wind farm measurements are available in literature. In this paper, harmonic emissions of an $18 \mathrm{MW}$ wind farm are investigated using extensive measurements, and the deterministic and stochastic characterization of wind farm harmonic currents is analyzed. Specific issues addressed in the paper include the harmonic variation with the wind farm operating point and the random characteristics of their magnitude and phase angle.
\end{abstract}

Index Terms-Harmonics, statistical analysis, wind power generation, wind turbines.

\section{INTRODUCTION}

$\mathbf{T}$ HE NUMBER of wind farms is increasing worldwide. In addition, the wind turbines (WTs) installed in these farms are over $1 \mathrm{MW}$. This poses power quality problems such as harmonic current emissions [1]-[8]. These, mainly caused by high-power converters with low switching frequency, imperfections in control systems, nonlinearities in generators and transformers, etc., provoke voltage distortion in networks, and their measurement and inclusion in WT power certificates are therefore required by current standards [8]-[10].

Knowledge of wind farm harmonic behavior is fundamental to study the influence of these farms on network harmonic distortion. Thus, the assessment of the wind farm harmonic spectrum and the analysis of the influence of the WT operating point on it are important issues in wind farm studies. Although WT harmonic emissions are a well-known topic, very few studies based on actual measurements have been published [2]-[6]. Since WT behavior stochastically varies with time, wind farm harmonic currents cannot be described by a deterministic assessment only. In addition, the random operating conditions of WTs require the application of probabilistic techniques to characterize wind farm harmonic currents correctly. There is a lack of studies on this topic in the literature and only references [1]-[3]

Manuscript received July 9, 2009; revised December 18, 2009 and January 30, 2010; accepted March 1, 2010. Date of publication June 3, 2010; date of current version November 19, 2010. This work was supported by the Grant DPI2006-2157. Paper no. TEC-00269-2009.

L. Sainz and J. J. Mesas are with the Department of Electrical Engineering, School of Industrial Engineering of Barcelona, Technical University of Catalonia, Barcelona 08028, Spain (e-mail: sainz@ee.upc.edu; juan.jose. mesas@upc.edu).

R. Teodorescu is with the Institute of Energy Technology (Power Electronics Section), Aalborg University, Aalborg East 9220, Denmark (e-mail: ret@iet.aau.dk).

P. Rodriguez is with the Department of Electrical Engineering, Escola Universitària d'Enginyeria Tècnica Industrial de Terrassa, Technical University of Catalonia, Terrassa 08222, Spain (e-mail: prodriguez@ee.upc.edu).

Digital Object Identifier 10.1109/TEC.2010.2045379 analyze the stochastic assessment of these currents. The former discusses the summation of random harmonic currents due to individual WTs and theoretically determines the probability density function (pdf) of the net harmonic current of wind farms. The latter characterizes the statistical behavior of WT harmonic current emissions from experimental measurements. On the other hand, there are many general studies on the pdfs of harmonic current phasors and the probability characteristics of their sum [11]-[13].

In this paper, the harmonic current behavior of an $18 \mathrm{MW}$ wind farm is investigated from a large number of measurements. The wind farm harmonic emissions are studied, and the influence of the wind farm working point on these emissions is analyzed. In addition, scatter plots of the harmonics (magnitude and phase angle) versus the wind farm output power are presented, and the random behavior of the harmonic emissions is analyzed from the experimental measurements. The pdfs of the harmonic magnitudes and phase angles are discussed and compared in detail with the analytical and empirical distributions in the literature. Finally, two pdfs are proposed to characterize the harmonic current stochastic behavior: Stacy distribution for the magnitudes and a combination of normal and uniform distributions, which is called normal-uniform distribution, for the phase angles.

\section{WIND FARM MEASUREMENTS}

\section{A. Studied Wind Farm}

The aim of the study is to investigate the harmonic current emissions of the wind farm in the one-line diagram of Fig. 1. The farm consists of $30 \times 600 \mathrm{~kW}$ WT squirrel-cage induction generators (SCIGs) connected to the medium voltage (MV) collector with 20/0.69 kV transformers. Although not shown in Fig. 1, power quality conditioners are generally connected to WTs to improve power quality. The rated power of the wind farm is $P_{N}=18 \mathrm{MW}$ and the rated current at the point of common coupling (PCC) is $I_{N}=522 \mathrm{~A}$. A $110 / 20 \mathrm{kV}$ transformer substation connects the MV and high voltage (HV) busbars.

The wind farm harmonic currents $\underline{i}_{h}=i_{h} \angle \phi_{h}=x_{h}+j y_{h}$ at the PCC depend on the sum of the individual WT harmonic currents $\underline{i}_{w t, h}=i_{w t, h} \angle \phi_{w t, h}=x_{w t, h}+j y_{w t, h}$, i.e.,

$$
\begin{aligned}
\underline{i}_{h} & =i_{h} \angle \phi_{h}=x_{h}+j y_{h}=\sum_{w t=1}^{N} x_{w t, h}+j \sum_{w t=1}^{N} y_{w t, h} \\
& =\sum_{w t=1}^{N} \underline{i}_{w t, h}
\end{aligned}
$$

where $N=30$ is the number of WT SCIGs in the wind farm. 


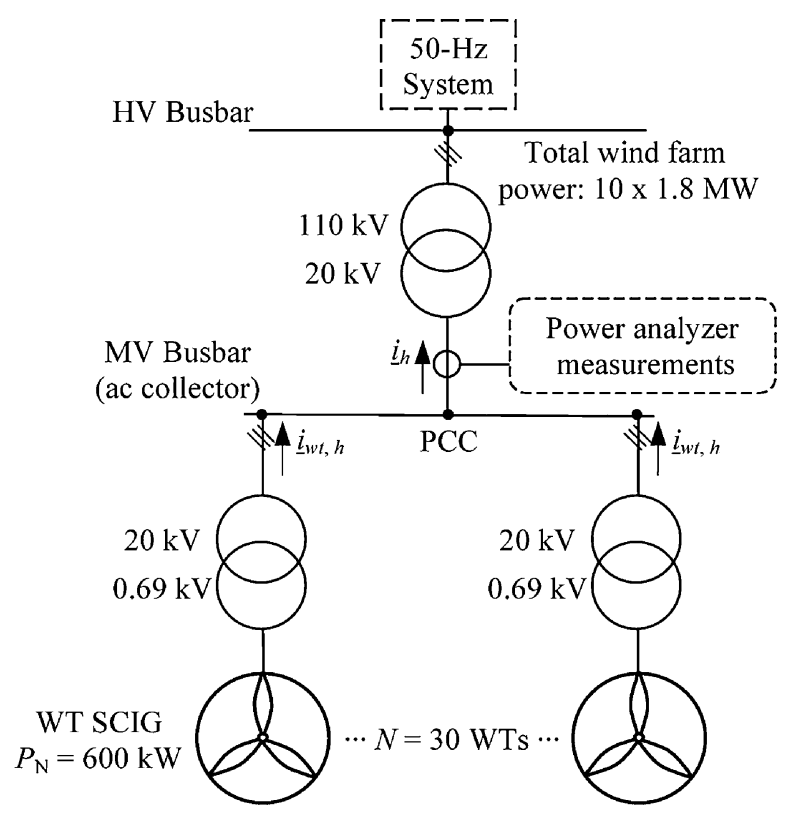

Fig. 1. Structure of the wind farm.

\section{B. Harmonic Current Measurements}

Measurements were carried out at the wind farm PCC (i.e., at the wind farm MV collector, Fig. 1) with a power network analyzer AR5-L of CIRCUTOR according to the requirements of IEC 61400-21 [9] and IEC 61000-4-7 [10] standards. The analyzer has a $0.5 \%$ voltage and current accuracy class, a sampling frequency sufficient to evaluate up to the 63rd harmonic order and a $1 \mathrm{Mb}$ on-board memory to save all measured or calculated parameters for future retrieving. The current clamps used in the measurements have a total full-scale accuracy of $1 \%$ with a bandwidth of $10 \mathrm{~Hz}$ to $5 \mathrm{kHz}$. Active and reactive powers, power factor, and voltage and current waveforms were recorded over a 6-day period with a 10-min time interval between readings, each record being the 10-min average value. The long storage period allowed obtaining data for the whole power range of the wind farm, and the 10-min average values provided sufficient accuracy of the voltage and current measurements to evaluate outstanding aspects of their harmonic behavior despite the unpredictability of wind conditions [2] and [9]. All the recorded voltage and current values were stored on a hard disk and treated with customized MATLAB software for harmonic analysis. Thus, assuming that the magnitude of the currents is stationary, the Fourier transform was applied to a 20-ms rectangular window, providing a $50-\mathrm{Hz}$ frequency resolution. The harmonics were evaluated up to the 15 order ( $2.5 \mathrm{kHz}$ for $50 \mathrm{~Hz}$ systems).

For space reasons, the paper summarizes the most representative results obtained to support the conclusions drawn.

\section{Deterministic Assessment OF THE Wind FARM HARMONIC CURRENTS}

\section{A. Current Harmonic Spectrum}

The measured wind farm output current and MV collector voltage waveforms are shown in Fig. 2(a), considering two

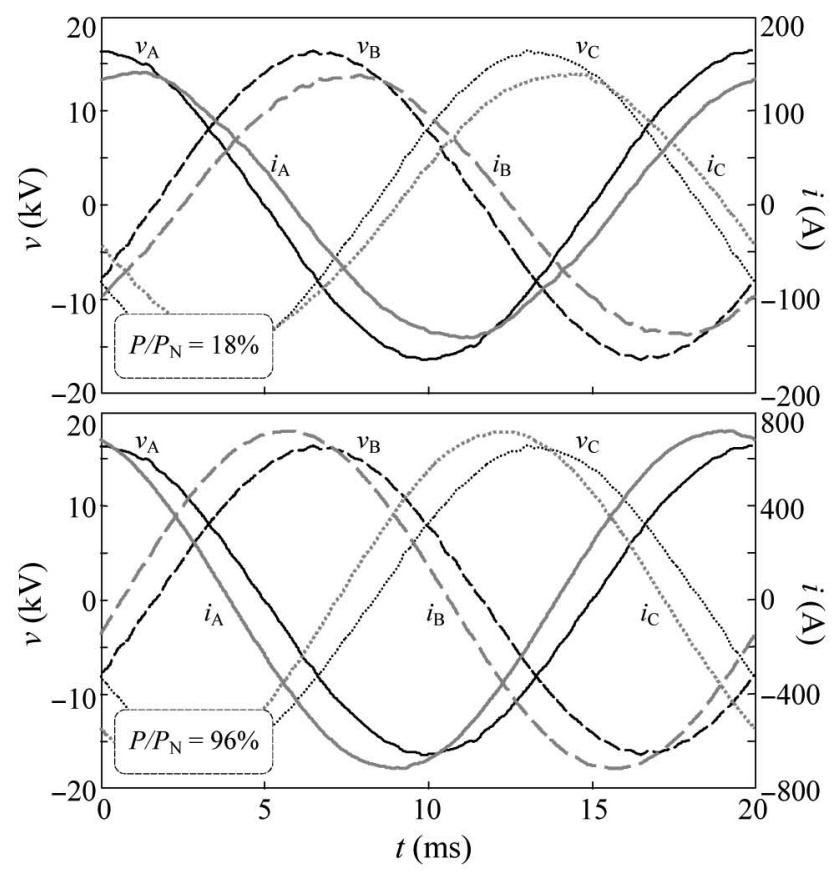

(a)

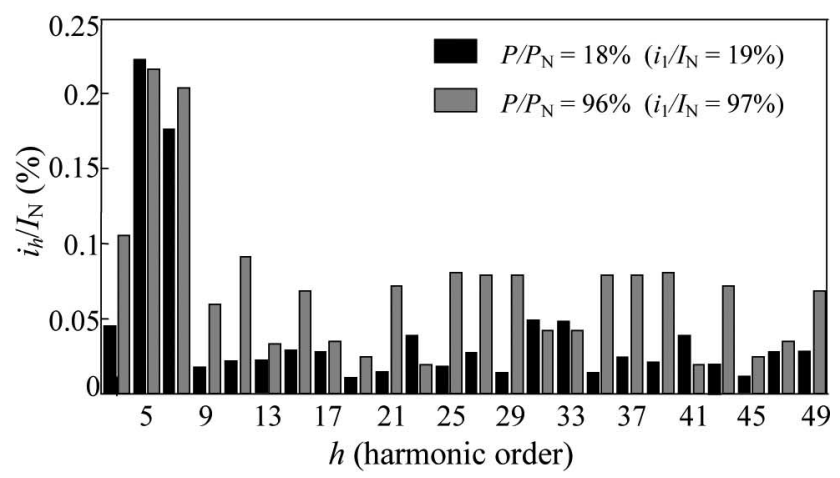

(b)

Fig. 2. Wind farm measurements at PCC for two power ratios: (a) voltage and output current waveforms. (b) Harmonic spectrum of phase A current.

wind farm power ratios (percentage of $P_{N}$ ). It was also numerically verified that the reactive power does not change significantly from lagging $\left(P / P_{N}=18 \%\right)$ to leading $\left(P / P_{N}=96 \%\right)$, and consequently the power factor changes from extreme values (outliers) of one to values close to one, respectively [see Fig. 2(a)]. Although the current waveforms are not perfectly balanced due to the unbalance of the MV collector voltages $v_{A}, v_{B}$, and $v_{C}$, the current unbalance is small enough to allow the study of the harmonic currents only from the phase A current. The voltage unbalance can be quantified from the unbalance factor of the MV collector voltages $m_{v}=v_{+} / v_{-}$, where $v_{+}$and $v_{-}$are the positive and negative sequence components of these voltages, which can be calculated from the Fortescue transformation [18]

$$
\left[\begin{array}{l}
\underline{v}_{0} \\
\underline{v}_{+} \\
\underline{v}_{-}
\end{array}\right]=\frac{1}{3}\left[\begin{array}{ccc}
1 & 1 & 1 \\
1 & a & a^{2} \\
1 & a^{2} & a
\end{array}\right] \cdot\left[\begin{array}{l}
\underline{v}_{\mathrm{A}} \\
\underline{v}_{\mathrm{B}} \\
\underline{v}_{\mathrm{C}}
\end{array}\right], \quad\left(a=e^{j 2 \pi / 3}\right) .
$$




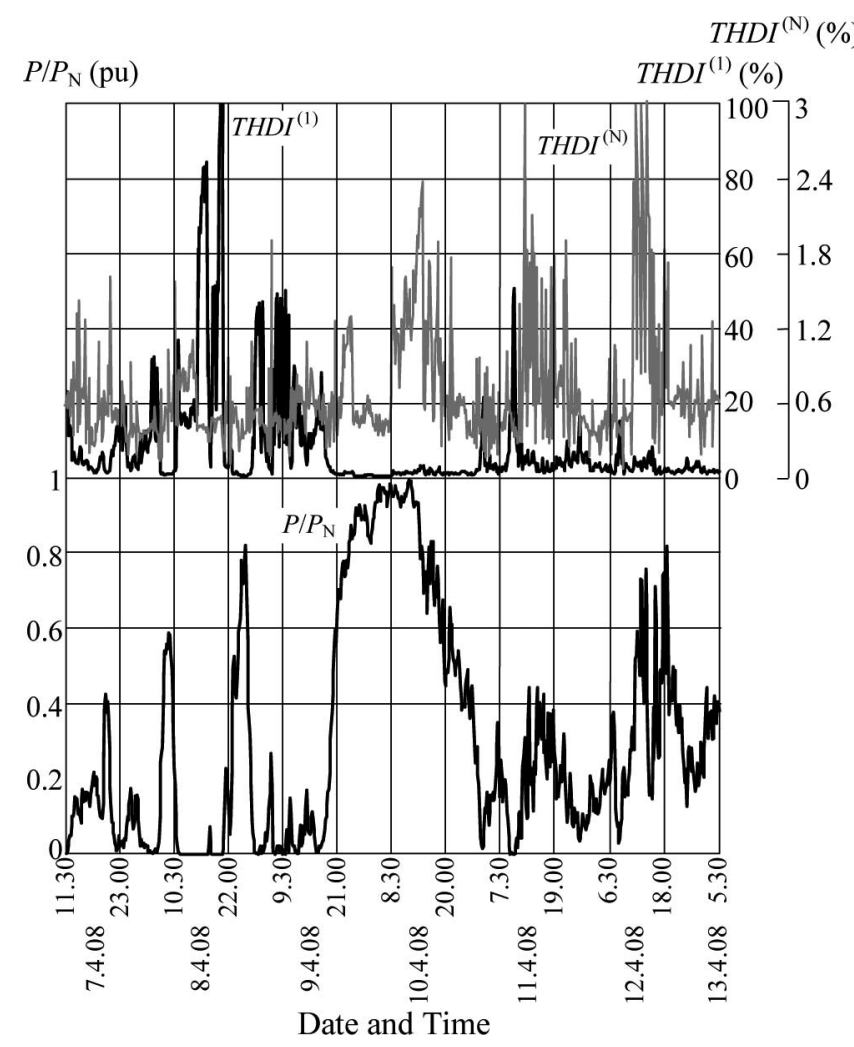

Fig. 3. Time course of the total harmonic distortion and output power ratio.

In the studied cases, $m_{v, 18 \%}=0.2 \%$ and $m_{v, 96 \%}=0.21 \%$. Thus, only the harmonic spectrum of the phase A current $\left(i_{h}\right.$ with $h=3,5 \ldots$ ) referred to the wind farm rated current $I_{N}$ is plotted in Fig. 2(b). The even harmonics were also measured and assessed, but are not included in the figure due to their negligible value. The following remarks on the aforementioned measurements can be made.

1) The dominant harmonics belong to the low-order set (in particular, the highest are fifth and seventh).

2) Low-order zero sequence harmonics, in particular third and ninth, also appear in the spectrum due to the unbalance of the MV collector voltages $v_{A}, v_{B}$, and $v_{C}$.

3) A high-order harmonic pattern can also be recognized in the current spectrum between 1.0 and $2.0 \mathrm{kHz}(21 \mathrm{st}-41 \mathrm{st}$ harmonics for $50 \mathrm{~Hz}$ systems).

\section{B. Effect of WT Operating Point on Harmonic Currents}

Harmonic current emission dependence on the WT operating point (i.e., wind speed conditions) is an important issue in wind farm studies.

To analyze this dependence, the time course of the total harmonic current distortion $(T H D I)$ and the wind farm output power is outlined in Fig. 3 for the measurement period. The total distortion is calculated from the recorded current waveforms according to IEC 61000-3-6 [17]

$$
\operatorname{THDI}^{(1)}=\frac{\sqrt{\sum_{k=2}^{50} i_{k}^{2}}}{i_{1}}
$$
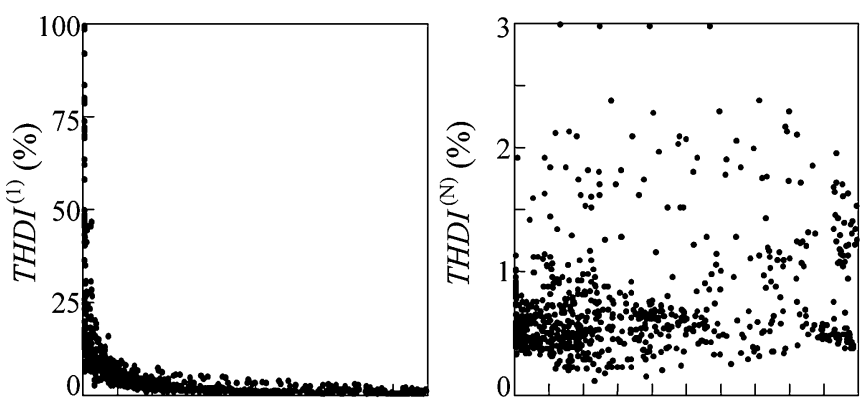

(a)
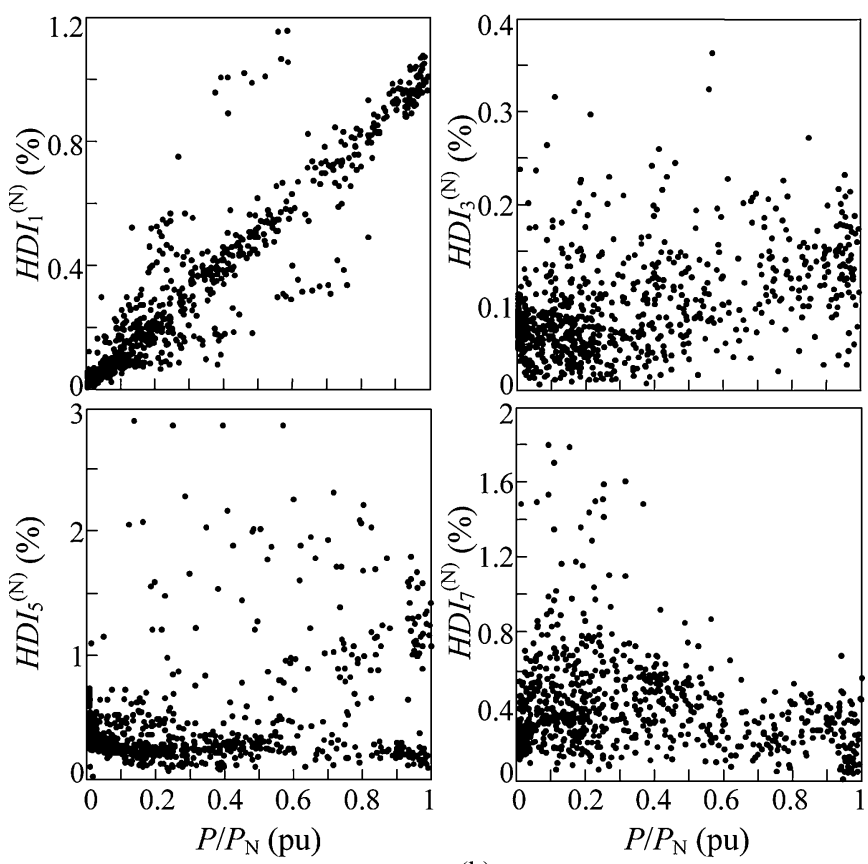

(b)

Fig. 4. Wind farm harmonic distortion versus output power for the total measurement period: (a) THDI referred to the fundamental component (left) and the rated value (right) of the WT farm current. (b) $H D I_{1}$ and $H D I_{h}$ referred to the rated value of the WT farm current.

where $i_{1}$ and $i_{k}$ are the fundamental and harmonic components of the wind farm output current at the PCC. As can be seen, the $T H D I^{(1)}$ course is characterized by high and low values under low and high generation conditions, respectively. This result is also summarized in Fig. 4(a), which shows the scatter plot of the $T H D I^{(1)}$ versus the output power from the measurement results of the whole investigated period. It is noted that the THDI ${ }^{(1)}$ value at the wind farm PCC falls exponentially with power output.

The harmonic distortion behavior observed in Fig. 4(a) is not due to the falling level of harmonic currents but to a rising value of the fundamental current. This becomes clear by analyzing the total and the individual harmonic current distortion, defined as [9]

$$
T H D I^{(\mathrm{N})}=\frac{\sqrt{\sum_{k=2}^{50} i_{k}^{2}}}{I_{N}} \quad H D I_{h}^{(\mathrm{N})}=\frac{i_{h}}{I_{N}}, \quad(h=1,3 \ldots)
$$




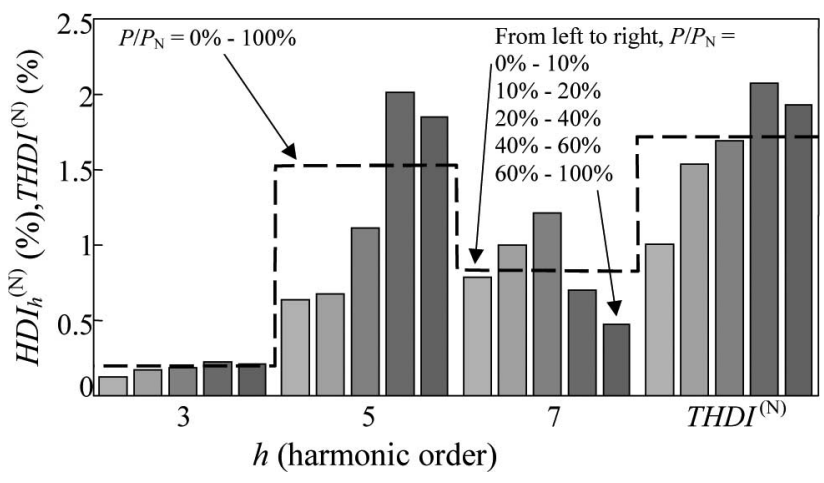

Fig. 5. Wind farm harmonic current emission (95\% values) for the whole range of power (dashed line) and for different loading conditions (bars).

where $I_{N}$ represents the wind farm rated current and $i_{h}$ the fundamental and harmonic components of the wind farm output current at the PCC. Thus, for the whole measurement period, the time course and the scatter plot of the $T H D I^{(N)}$ are shown in Figs. 3 and 4(a), respectively, and the scatter plots of $H D I_{h}^{(N)}$ of the first, third, fifth, and seventh harmonics are shown in Fig. 4(b). From these figures, the following can be noted.

1) The fundamental current increases proportionally with the wind farm output power while the harmonic currents vary stochastically.

2) The fifth and seventh harmonics are the dominant ones, and the total harmonic distortion depends mainly on them.

3) In spite of the random behavior of the wind farm harmonic currents and based on their small variation with respect to the wind farm operating point, for harmonic load flow purposes these currents are usually modeled as fixed current injections of given amplitudes per frequency. In accordance with IEC 61000 standards, the magnitudes adopted for harmonic current injections are generally the 95\% nonexceeding probability values of these currents. Thus, Fig. 5 shows the third, fifth, and seventh harmonic current values for the whole range (dashed line) and for different ranges (bar plot) of power. It is observed that fixed current injection modeling must be taken with care because the $95 \%$ nonexceeding probability values clearly depend on the power level.

To consider the summation effects of different harmonic sources (e.g., wind farms), besides the magnitudes of these sources, their phase angles are required. Thus, Fig. 6 shows the phase angle scatter plots of the most significant harmonic currents (i.e., fifth and seventh harmonics) versus the output power of the whole investigated period. The random behavior of these angles, which is also true for the other harmonics, is worth noting. Although this behavior is usually considered as uniformly distributed over the interval $\left[0^{\circ}, 360^{\circ}\right)$ in the literature, Fig. 6 reveals that it must be analyzed in more detail.

From the earlier analysis, the following observations about the consideration of wind farms in harmonic penetration studies based on the current injection method can be made.

1) Deterministic models are usually based on the arithmetical sum of the average or the $95 \%$ values of the measured wind
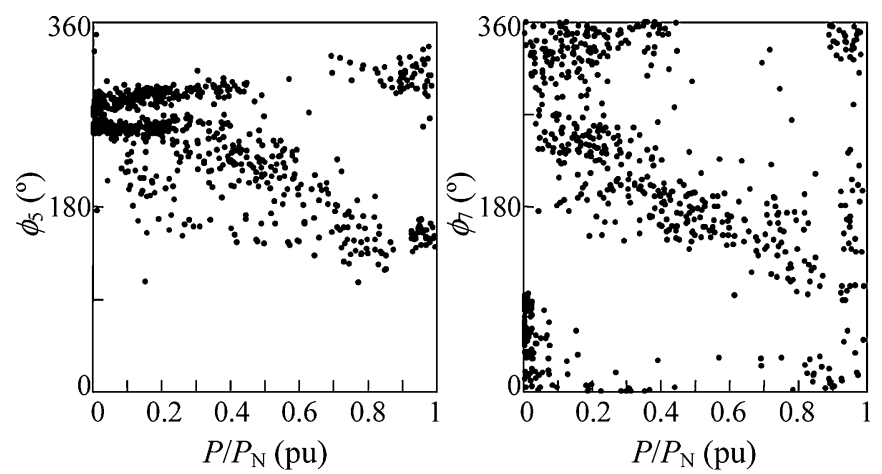

Fig. 6. Phase angles of the wind farm fifth and seventh harmonic currents.

farm harmonic currents. These models are the simplest ones and compensate for the lack of information about harmonic current phase angles. Nevertheless, they lead to overestimation of system harmonic distortion because the random variation of harmonics (in particular their phase angles) is disregarded.

2) At present, wind farm harmonic currents are modeled as random variables characterized by their pdfs. These models are the most realistic ones but their determination is not a simple task due to the complex random behavior of WTs and the shortage of data.

In the next section, the most significant harmonic currents injected by the wind farm are stochastically assessed from field measurements.

\section{STOCHASTIC ASSESSMENT OF WIND FARM HARMONIC CURRENTS}

In general, it is necessary to apply probabilistic techniques to evaluate the harmonic currents of wind farms because the operating conditions of WTs vary stochastically with time. This is reflected by the experimental results in Figs. 4(b) and 6. The simplest procedure to obtain the pdfs of wind farm currents is the Monte Carlo method if the pdfs of the individual WT harmonic currents are assumed known. Nevertheless, this method does not provide closed-form solutions. For this reason, several studies have been conducted to obtain analytical pdf expressions. If no fully analytical solution can be reached, sometimes there exist empirical solutions, which allow the determination of closed-forms equaling some statistical moments of actual and approximate distributions [12], [14]. In this section, the third, fifth, and seventh harmonic distributions obtained from wind farm experimental measurements are discussed and compared with some of the analytical and empirical distributions in the literature. Moreover, two pdfs are proposed to characterize the stochastic behavior of the harmonic current magnitudes and phase angles. In the statistical study, uppercase letters indicate random variables (e.g., $X_{h}, Y_{h}, I_{h}$, and $\Phi_{h}$ ) while lowercase letters represent the specific values in the variables (e.g., $x_{h}, y_{h}$, $i_{h}$, and $\phi_{h}$ ).

The harmonic current emissions of the wind farm depend on the statistical characteristics of the individual WT harmonic currents (i.e., the pdfs of their magnitudes and phase angles $I_{w t, h}$ 

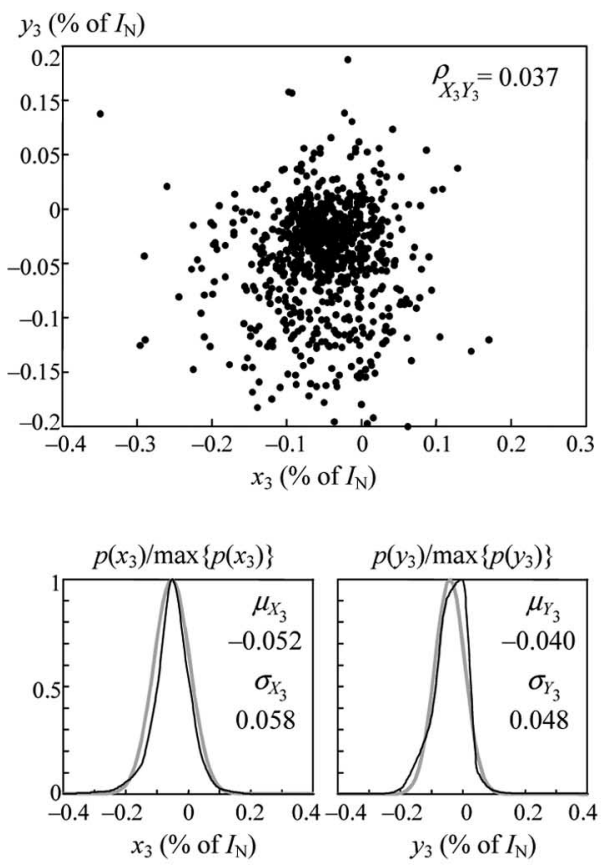

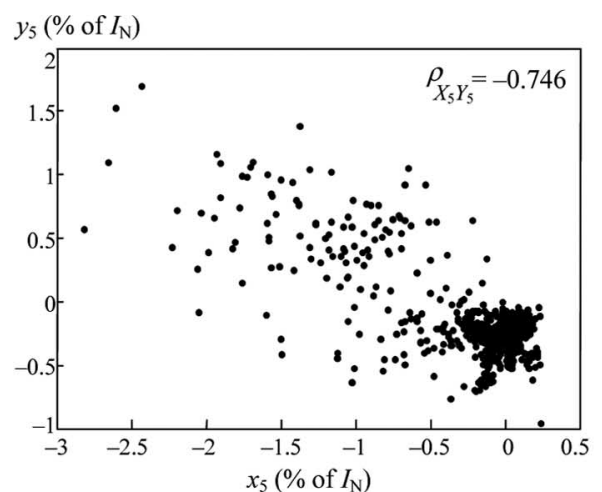

(a)

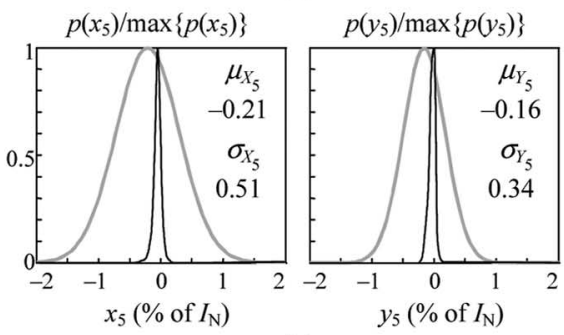

(b)
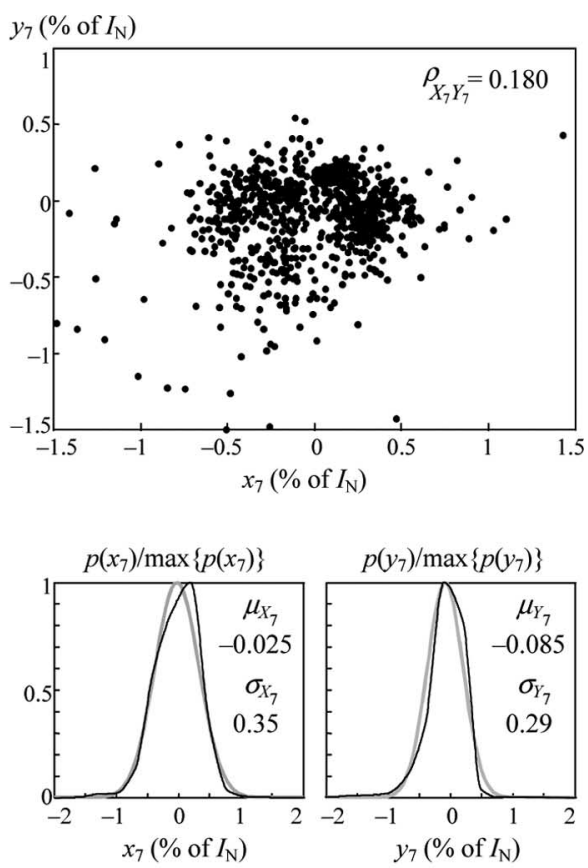

Fig. 7. Statistical analysis of the harmonic current $x-y$ projections: (a) scatter plots. (b) pdfs [black line: actual distributions; gray line: normal distributions $\left.N\left(\mu_{(\cdot)}, \sigma_{(\cdot)}^{2}\right)\right]$.

and $\Phi_{w t, h}$ or their real and imaginary components $X_{w t, h}$ and $\left.Y_{w t, h}\right)$. However, considering the WT harmonic currents $\underline{i}_{w t, h}$ statistically independent of each other [1], the central limit theorem states that the pdfs of $X_{h}$ and $Y_{h}$ in (1) can be approximated by the normal distributions $N\left(\mu_{X_{h}}, \sigma_{X_{h}}^{2}\right)$ and $N\left(\mu_{Y_{h}}, \sigma_{Y_{h}}^{2}\right)$ regardless of the individual variable distributions if the number of WTs is sufficiently large [11], [12], and [14]. The mean value and the standard deviation of the aforementioned variables are given by the following:

$$
\mu_{U_{h}}=\sum_{w t=1}^{N} \mu_{U_{w t, h}} \quad \sigma_{U_{h}}^{2}=\sum_{w t=1}^{N} \sigma_{U_{w t, h}}^{2} \quad U=X, Y .
$$

According to that and considering $\rho_{X_{h} Y_{h}}$ the correlation coefficient of the variables, the joint pdf of $X_{h}$ and $Y_{h}$ in (1) is a bivariate normal distribution [11], [12], [14], i.e.,

$$
p_{X_{h} Y_{h}}\left(x_{h}, y_{h}\right)=\frac{\exp \left(-T /\left[2\left(1-\rho_{X_{h} Y_{h}}^{2}\right)\right]\right)}{2 \pi \sigma_{X_{h}} \sigma_{Y_{h}} \sqrt{1-\rho_{X_{h} Y_{h}}^{2}}}
$$

where

$$
\begin{aligned}
T= & \frac{\left(x_{h}-\mu_{X_{h}}\right)^{2}}{\sigma_{X_{h}}^{2}}-\frac{2 \rho_{X_{h} Y_{h}}\left(x_{h}-\mu_{X_{h}}\right)\left(y_{h}-\mu_{Y_{h}}\right)}{\sigma_{X_{h}} \sigma_{Y_{h}}} \\
& +\frac{\left(y_{h}-\mu_{Y_{h}}\right)^{2}}{\sigma_{Y_{h}}^{2}} .
\end{aligned}
$$

The correlation coefficient ranges between \pm 1 , i.e., $\left|\rho_{X_{h} Y_{h}}\right| \leq 1$. It is a measure of linear dependence between the variables, and $\rho_{X_{h} Y_{h}}= \pm 1$ implies variables perfectly correlated on a straight line with positive/negative slope [11], [12], [14].
Thus, considering (6), the pdfs of the harmonic magnitudes and phase angles can be directly derived from the following relations [11], [12]:

$$
\begin{aligned}
p_{I_{h}}\left(i_{h}\right) & =\int_{0}^{2 \pi} i_{h} p_{X_{h} Y_{h}}\left(x_{h}, y_{h}\right) d \phi_{h} \\
p_{\Phi_{h}}\left(\phi_{h}\right) & =\int_{0}^{\infty} \phi_{h} p_{X_{h} Y_{h}}\left(x_{h}, y_{h}\right) d i_{h} .
\end{aligned}
$$

The difficulty in analytically solving the aforementioned integrals depends on the correlation coefficient, mean, and standard deviation values of the $X_{h}$ and $Y_{h}$ projections [12]. If $\rho_{X_{h} Y_{h}}=0$ and the variables are jointly normal as in the studied case, these variables are independent (in general, $\rho_{X_{h} Y_{h}}=0$ implies linear independence of the random variables but not absolute independence) [14], and the integrals (8) are relatively easy to handle [12]. The conditions $\mu_{X_{h}}$ or $\mu_{Y_{h}}$ equal to zero, and $\sigma_{X_{h}}=\sigma_{Y_{h}}$ are also useful to facilitate the solution of the integrals [12].

Considering the aforementioned comments and before studying the distributions of the harmonic current magnitudes and phase angles, the random behavior of the third, fifth, and seventh harmonic current $X_{h}$ and $Y_{h}$ components is analyzed. Thus, their scatter plots and pdfs are shown in Fig. 7. The $\rho_{X_{h} Y_{h}}, \mu_{(\cdot)}$, and $\sigma_{(\cdot)}$ values obtained from experimental measurements are also labeled in this figure. It can be observed that the $X_{h}$ and $Y_{h}$ components of the third and seventh harmonics have small correlation coefficient and mean and similar standard deviation. Moreover, their pdfs can be approximated by a normal distribution. On the other hand, the fifth harmonic $X_{h}$ and $Y_{h}$ components have a correlation coefficient close to -1 due to the 

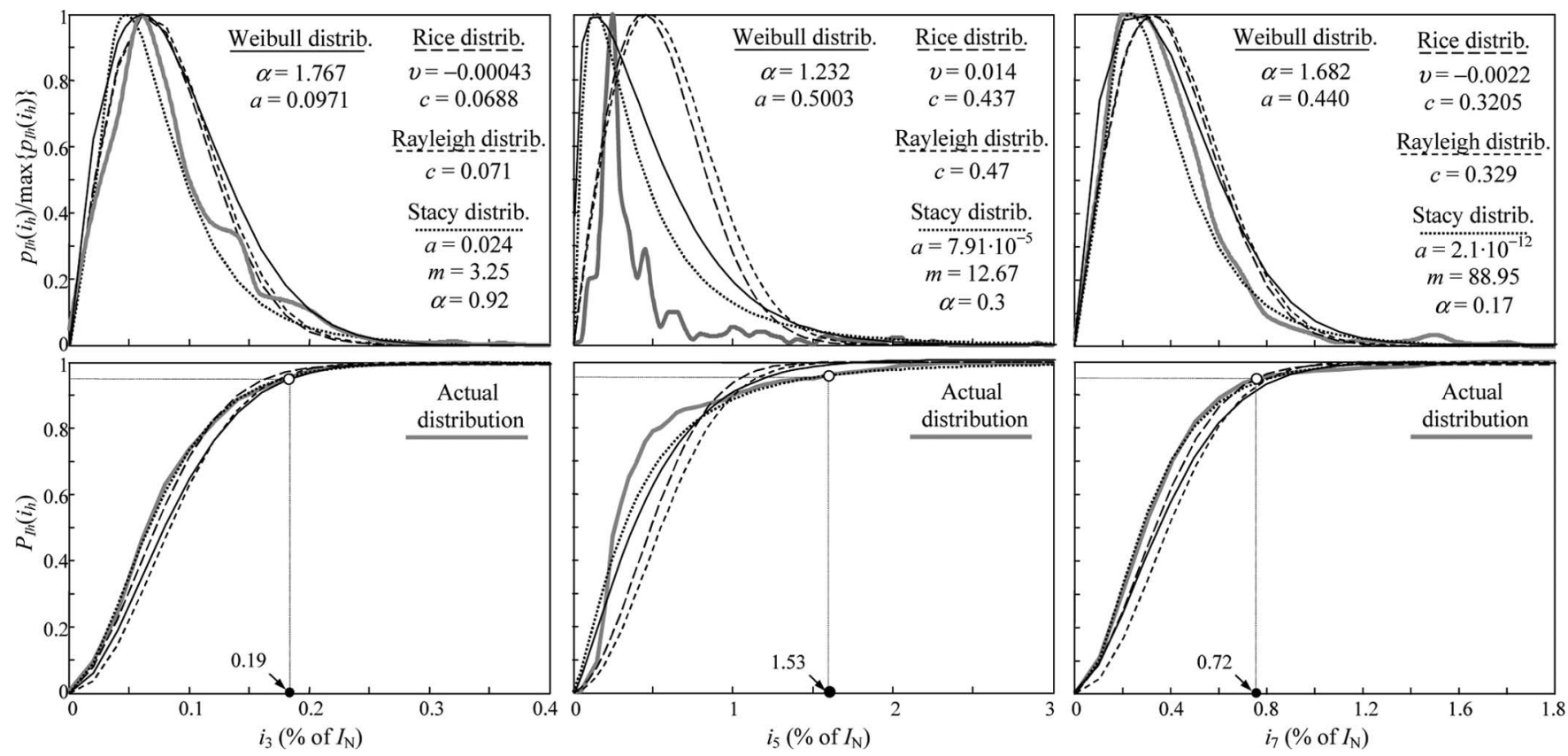

Fig. 8. Pdf (up) and probability distribution function (down) of the third, fifth, and seventh harmonic current magnitudes.

negative linear dependence, as shown in Fig. 7, and their pdfs cannot be approximated by a normal distribution.

\section{A. Magnitude Distribution}

Fig. 8 shows the pdfs and probability distribution functions of the third, fifth, and seventh harmonic current magnitudes obtained from their experimental measurements (gray lines). The 95\% values of the harmonic currents (dashed lines in Fig. 5) are also labeled in Fig. 8.

In the literature, the WT power quality assessment standard IEC 61400-21 [9] does not provide any information about the pdfs of these harmonic emissions and only recommends the summation rule of WT harmonic currents

$$
i_{h}=\sqrt[\beta]{\sum_{w t=1}^{N}\left(i_{w t, h}\right)^{\beta}}, \quad\left(\begin{array}{c}
\beta=1 \\
\beta=1.4 \text { for } \quad h<5 \\
\text { for } 5 \leq h \leq 10
\end{array}\right) .
$$

Reference [1] proposes wind farm harmonic magnitude pdfs from the theoretical analysis of the summation of WT harmonics in wind farms. Based on [11] and [12], the study assumes two simplified distributions of the WT harmonic current phasors that verify the hypotheses of $\rho_{X_{h} Y_{h}}=0, \mu_{X_{h}}=\mu_{Y_{h}}=0$, and $\sigma_{X_{h}}=\sigma_{Y_{h}}=\sigma$. Thus, considering these distributions and the central limit theorem approach (6), the integration of (8) becomes the Rayleigh distribution [12]

$$
p_{I_{h}}\left(i_{h} ; c\right)=\left(\frac{i_{h}}{c^{2}}\right) \exp \left(-\frac{i_{h}^{2}}{2 c^{2}}\right)
$$

where $c=\sigma$. Moreover, the summation rule (9) proposed in [9] for the wind farm harmonic current magnitude calculation is also deduced from (10) in [1].
According to [12], a slightly more general distribution than (10) called Rice distribution can be proposed if the mean value hypothesis is relaxed, and only one of the means is considered zero (i.e., $\mu_{X_{h}}=0$ or $\mu_{Y_{h}}=0$ )

$$
p_{I_{h}}\left(i_{h} ; c, v\right)=\left(\frac{i_{h}}{c^{2}}\right) \exp \left(-\frac{i_{h}^{2}+v^{2}}{2 c^{2}}\right) I_{0}\left(\frac{i_{h} v}{c^{2}}\right)
$$

where $I_{0}(\cdot)$ is the modified Bessel function of the first kind with order zero, $v=\mu_{Y_{h}}\left(\right.$ or $\mu_{X_{h}}$ ) and $c=\sigma$.

Thus, both distributions were fitted to the experimental measurements of the third, fifth, and seventh harmonics. The results are compared with the actual distributions in Fig. 8. It is observed that the analytical distributions of the third and seventh harmonics closely agree with the actual ones due to the random characteristics of their $X_{h}$ and $Y_{h}$ components. Also note that the Rice distribution is the best because the $X_{h}$ and $Y_{h}$ data mean values are not zero, as shown in Fig. 7. This is not true for the fifth harmonic as its components do not follow a normal distribution and their correlation coefficient is not zero. These results show that the theoretical assumptions in [1] about WT harmonic current distributions for the stochastic assessment of wind farm harmonic current emissions are questionable and must be therefore carefully analyzed from the experimental measurements in actual installations. These conclusions are confirmed in [2], where the harmonic emissions of WTs are experimentally investigated and their stochastic behavior is characterized. Thus, the phase angles of the low- and high-order harmonics are experimentally approximated by normal and uniform distributions, respectively, and the magnitudes of the low-order harmonics are experimentally approximated by 


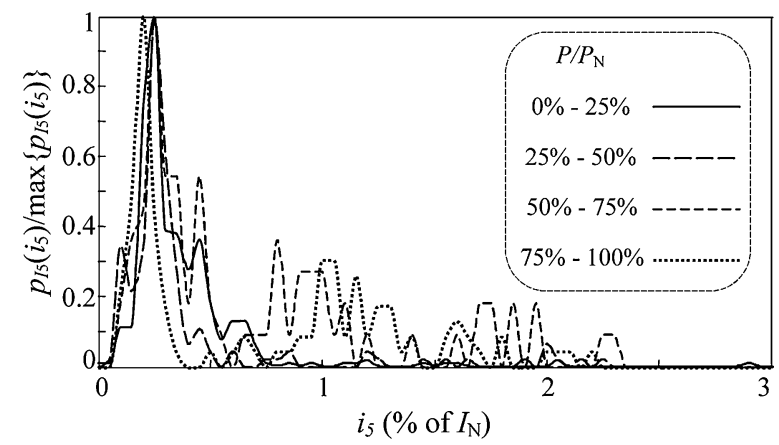

Fig. 9. Pdfs of the fifth harmonic current magnitude at different operating points.

the Weibull distribution [2], [14]

$$
p_{I_{h}}\left(i_{h} ; a, \alpha\right)=\frac{\alpha}{a}\left(\frac{i_{h}}{a}\right)^{\alpha-1} \exp \left(-\left(\frac{i_{h}}{a}\right)^{\alpha}\right)
$$

which is more general than the Rayleigh distribution because (10) can be obtained from (12) by setting $\alpha=2$ and $a=$ $\sqrt{ } 2 c$. Unfortunately, no analytical expressions for the magnitude stochastic characterization of wind farm emissions can be deduced from the WT distributions in [2]. However, the Monte Carlo simulations numerically confirmed that, considering these experimental distributions, the pdfs of the wind farm harmonic magnitudes could also be closely approximated by the Weibull distribution (12). Thus, this distribution was fitted to the experimental measurements of the third, fifth, and seventh harmonics, see Fig. 8. Note that it is better than the Rayleigh and Rice distributions but the fifth harmonic results are still not satisfactory.

In this situation, some empirical methods use a not fully analytical demonstration to obtain closed forms of the investigated distributions [12], [13]. Thus, an empirical solution based on a generalization of the Gamma distribution presented by Stacy in [15] and Stacy and Mihram [16] is suggested

$$
p_{I_{h}}\left(i_{h} ; a, m, \alpha\right)=\frac{\alpha i_{h}^{\alpha m-1}}{\Gamma(m) a^{\alpha m}} \exp \left[-\left(\frac{i_{h}}{a}\right)^{\alpha}\right]
$$

where $\Gamma(\cdot)$ is the Gamma function and $a, m, \alpha>0$. This distribution offers a large variety of shapes and includes several distributions, see Appendix A. A particular case of (13) with $\alpha=2$ for empirical characterization of harmonic magnitude distributions in general cases is proposed in [12] and [13]. Moreover, its moments appear in simple closed-form expressions, which can be used to better adjust the experimental measurement distribution. The Stacy distribution was fitted to the experimental measurements of the third, fifth, and seventh harmonics. The obtained results are compared with the actual distributions in Fig. 8. It must be noted that the proposed empirical distribution closely agrees with the actual ones even for the fifth harmonic.

Since the influence of the wind farm operating point on harmonic currents is small (see comments on Figs. 3 and 4 in Section III-B), the pdfs in Fig. 8 do not change significantly from one power level to another. To verify this, Fig. 9 provides the pdfs of the fifth harmonic current at four different power levels. It was checked that the results for the fifth harmonic in Fig. 9 are also true for the third and seventh harmonics.

These distributions were fitted to the actual ones by the method of moments [14], which is briefly described in Appendix B.

\section{B. Phase Angle Distribution}

Harmonic current phase angles are not required in wind farm studies [9], but they are important for the calculation of harmonic current summations in harmonic penetration analysis. Thus, Fig. 10 shows the pdfs of the third, fifth, and seventh harmonic current phase angles obtained from their experimental measurements.

Considering the two simplified distributions for WT harmonic currents in [1], the phase angle pdfs could be fitted with the uniform distribution from $0^{\circ}$ to $360^{\circ}$, i.e., $\Phi_{h} \sim$ $U\left(0^{\circ}, 360^{\circ}\right)$. Nevertheless, as commented for the magnitudes in Section IV-A, this theoretical result, which is the only one available in the literature to the knowledge of the authors, is questionable and must therefore be carefully analyzed from experimental measurements. Thus, considering the experimental distributions for WT harmonic currents in [2], the Monte Carlo method numerically confirmed that the pdfs of the wind farm harmonic phase angles could be closely fitted with the normal and uniform distributions for low- and high-order harmonics, respectively.

It is observed that the third and fifth harmonic phase angles have a normal-type distribution (one and two distinct normaltype peaks, respectively) whereas the seventh harmonic phase angle tends to a uniform-type distribution over the whole $\left[0^{\circ}\right.$, $360^{\circ}$ ) interval. It was verified that the phase angles of harmonics higher than seventh also exhibit uniform-type distributions. Considering this and applying the total probability theorem [14], the following pdf, which is called normal-uniform distribution, is proposed to describe the stochastic behavior of wind farm harmonic current phase angles

$$
\begin{aligned}
& p_{\Phi_{h}}\left(\phi_{h} ; d_{1}, \mu_{1}, \sigma_{1}^{2}, d_{2}, \mu_{2}, \sigma_{2}^{2}\right)=d_{1} N\left(\phi_{h} ; \mu_{1}, \sigma_{1}^{2}\right) \\
& \quad+d_{2} N\left(\phi_{h} ; \mu_{2}, \sigma_{2}^{2}\right)+d_{3} U\left(\phi_{h} ; l b, u b\right)
\end{aligned}
$$

where $N(\mu, \sigma)$ is the normal distribution, $U(l b, u b)$ is the uniform distribution, $l b=0^{\circ}$ (lower boundary), $u b=360^{\circ}$ (upper boundary), and $d_{1}, d_{2}$, and $d_{3}$ are weighting factors verifying that $d_{1}+d_{2}+d_{3}=1$ (i.e., $d_{3}=1-d_{1}-d_{2}$ ) and $0 \leq d_{i} \leq 1$ $(i=1,2,3)$. The normal-uniform distribution was fitted to the experimental measurements. The obtained results are compared with the actual distributions in Fig. 10. It must be noted that the proposed distribution gives a correct result. This distribution was fitted to the actual ones by the method of moments [14], which is briefly described in Appendix B.

\section{CONCLUSION}

In this paper, the harmonic emissions of a $30 \times 600 \mathrm{~kW}$ WT SCIG wind farm are analyzed using field measurements to contribute to the understanding of wind farm harmonic behavior. The following conclusions can be drawn from the investigation. 

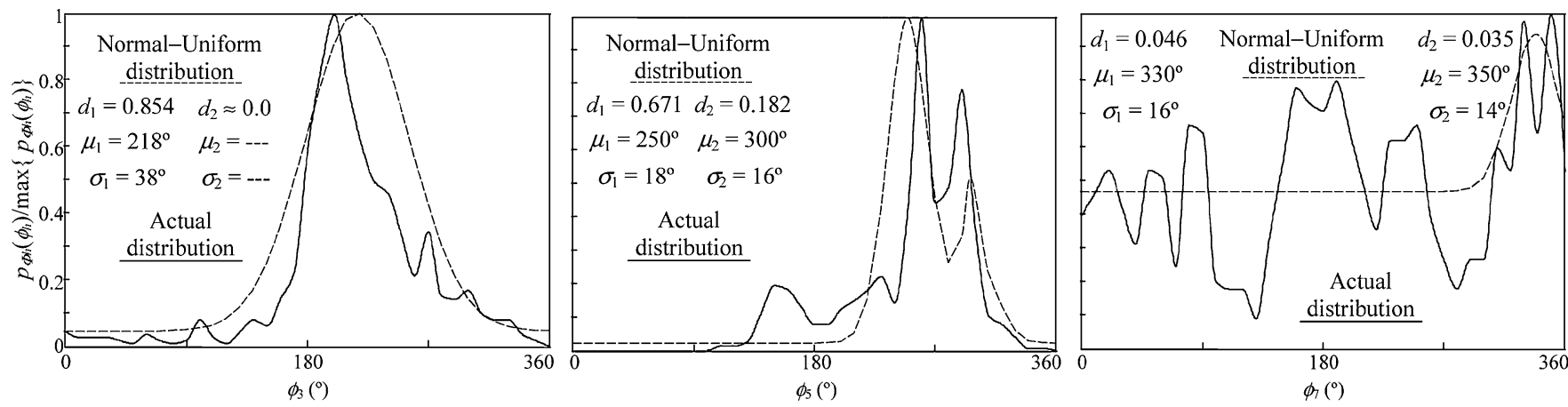

Fig. 10. Pdfs of the third, fifth, and seventh harmonic current phase angles.

TABLE I

Special Cases of the Stacy Distribution (ObTained From [12])

\begin{tabular}{cc}
\hline \hline Type & Probability density function \\
\hline Exponential $($ Exp $)$ & $f_{U}(u ; a, 1,1)$ \\
Gamma $($ Gam $)$ & $f_{U}(u ; a, m, 1)$ \\
Nakagami-m $(N a k)$ & $f_{U}(u ; a, m, 2)$ \\
Weibull $($ Weib $)$ & $f_{U}(u ; a, 1, \alpha)$ \\
Chi Squared $($ Chi 2$)$ & $f_{U}(u ; 2, n / 2,1), n$ degrees of freedom \\
Chi $($ Chi $)$ & $f_{U}(u ; \sqrt{2}, n / 2,2), n$ degrees of freedom \\
Half-Normal $(H N)$ & $f_{U}(u ; \sqrt{ } 2,1 / 2,2)$ \\
Circular Normal $(C N)$ & $f_{U}(u ; \sqrt{ } 2,1,2)$ \\
Spherical Normal $(S N)$ & $f_{U}(u ; \sqrt{ } 2,3 / 2,2)$ \\
Rayleigh $($ Ray $)$ & $f_{U}(u ; \sqrt{ } 2 \cdot \mathrm{c}, 1,2), \mathrm{c}>0$ \\
\hline \hline
\end{tabular}

1) Low-order characteristic harmonics are the dominant ones in the analyzed measurements.

2) A high-order harmonic pattern can also be recognized in the wind farm harmonic spectrum.

3) The harmonic distortion shows a clear random behavior despite the small influence of the wind farm operating point on it.

The paper also discusses the analytical and empirical distributions of wind farm harmonic currents in the literature, compares these distributions with those obtained from experimental measurements, and proposes pdf closed forms of wind farm harmonic currents. Thus, the Stacy and the normal-uniform distributions are used to statistically characterize the current magnitudes and phase angles, respectively. This is useful for statistically studying network harmonic distortion in the presence of wind farms and for analyzing the cancellation effect due to the harmonic current phase angle diversity on net harmonic currents injected by wind farms.

\section{APPENDIX A}

\section{Special Cases of the Stacy Distribution}

The pdfs included in the Stacy distribution are presented in Table I, and Fig. 11 provides some examples of these distributions.

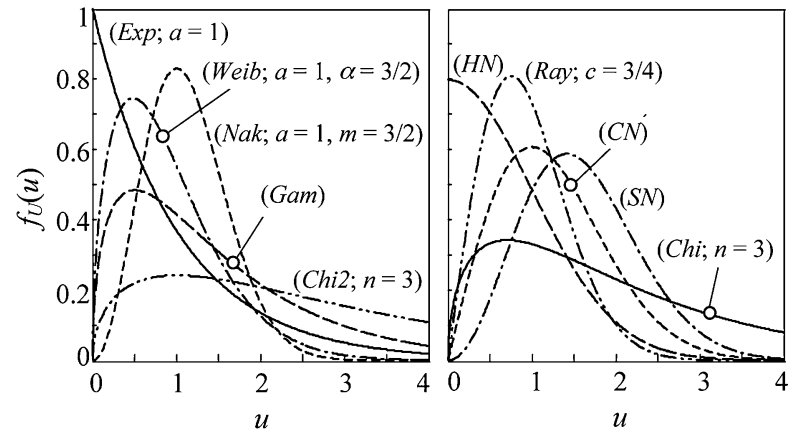

Fig. 11. Special cases of the Stacy distribution.

\section{APPENDIX B}

\section{ANALYTICAL PDF FitTing to ACTUAL DistRibutions}

To fit an analytical pdf $g_{Z}\left(z ; \kappa_{1}, \ldots, \kappa_{m}\right)$ to an actual distribution, the $g_{Z}$ parameters $\kappa_{1}$ to $\kappa_{m}$ must be estimated from the independently observed data $z_{1}, \ldots, z_{n}$ of the actual distribution [14].

Thus, considering

$\delta_{i}\left(\kappa_{1}, \ldots, \kappa_{m}\right)=E\left(Z^{i}\right)=\int_{-\infty}^{\infty} z^{n} g_{Z}(z) d z, \quad i=1,2, \ldots$

the $i$ th theoretical or population moment of $Z$, and

$$
D_{i}=\frac{1}{n} \sum_{j=1}^{n} z_{j}^{i}, \quad i=1,2, \ldots
$$

the $i$ th sample moment of the observed data, the estimation of the parameters $\kappa_{1}$ to $\kappa_{m}$ can be performed by the method of moments, which consists in equating a sufficient number of sample moments to the population ones to determine estimators $\kappa_{1, e}$ to $\kappa_{m, e}$ of the parameters, i.e.,

$$
\delta_{i}\left(\kappa_{1, \mathrm{e}}, \ldots, \kappa_{m, \mathrm{e}}\right)=D_{i}, \quad i=1,2, \ldots m .
$$

Although low-order moment equations are preferred because they require less manipulation of observed data, any convenient set of $m$ equations is sufficient to obtain $m$ estimators. The Rice, 
TABLE II

THEORETICAL MOMENTS OF THE NORMAL DISTRIBUTION

\begin{tabular}{cl}
\hline \hline Order & \multicolumn{1}{c}{ Moments, $M_{i}(\mu, \sigma)$} \\
\hline 1 & $\mu$ \\
2 & $\mu^{2}+\sigma^{2}$ \\
3 & $\mu^{3}+3 \mu \sigma^{2}$ \\
4 & $\mu^{4}+6 \mu^{2} \sigma^{2}+3 \sigma^{4}$ \\
5 & $\mu^{5}+10 \mu^{3} \sigma^{2}+15 \mu \sigma^{4}$ \\
6 & $\mu^{6}+15 \mu^{4} \sigma^{2}+45 \mu^{2} \sigma^{4}+15 \sigma^{6}$ \\
7 & $\mu^{7}+21 \mu^{5} \sigma^{2}+105 \mu^{3} \sigma^{4}+105 \mu \sigma^{6}$ \\
\hline \hline
\end{tabular}

Rayleigh, and Stacy distribution fittings are presented shortly according to this procedure.

The Rice distribution is fitted with the following moment equations:

$$
\begin{aligned}
& \delta_{1}\left(c_{e}, v_{e}\right)=c_{e} \sqrt{\frac{\pi}{2}} L_{1 / 2}\left(-\frac{v_{e}^{2}}{2 c_{e}^{2}}\right)=D_{1} \\
& \delta_{2}\left(c_{e}, v_{e}\right)=2 c_{e}^{2}+v_{e}^{2}=D_{2}
\end{aligned}
$$

where $L_{1 / 2}(u)$ denotes Laguerre polynomial

$$
L_{1 / 2}(u)=\exp (u / 2)\left((1-u) I_{0}(-u / 2)-u I_{1}(-u / 2)\right)
$$

and $I_{\eta}(\cdot)$ is the modified Bessel function of the first kind of order $\eta$.

The Weibull distribution is fitted with the following moment equation:

$$
\delta_{i}\left(a_{e}, \alpha_{e}\right)=a_{e}^{i} \Gamma\left(1+i / \alpha_{e}\right)=D_{i}, \quad i=1,2
$$

The Rayleigh distribution is fitted with the following moment equation:

$$
\delta_{2}\left(c_{e}\right)=2 c_{e}^{2}=D_{2} \quad \Rightarrow \quad c_{e}=\sqrt{0.5 D_{2}} .
$$

The Stacy distribution is fitted with the following moment equations:

$$
\delta_{i}\left(a_{e}, m_{e}, \alpha_{e}\right)=a_{e}^{i} \frac{\Gamma\left(m_{e}+i / \alpha_{e}\right)}{\Gamma\left(m_{e}\right)}=D_{i}, \quad i=1,2
$$

and

$$
a_{e}=\sqrt[\alpha_{e}]{\frac{E\left(Z^{\alpha_{e}}\right)}{m_{e}}} .
$$

A different procedure to fit the Stacy distribution is proposed in [16].
And the normal-uniform distribution is fitted with the following moment equations:

$$
\begin{gathered}
\delta_{i}\left(d_{1 e}, \mu_{1 e}, \sigma_{1 e}, d_{2 e}, \mu_{2 e}, \sigma_{2 e}\right)=d_{1 e} M_{i}\left(\mu_{1 e}, \sigma_{1 e}\right) \\
+d_{2 e} M_{i}\left(\mu_{2 e}, \sigma_{2 e}\right)+\left(1-d_{1 e}-d_{2 e}\right) \frac{1}{i+1} \sum_{k=0}^{i} l b^{k} u b^{k-i}=D_{i} \\
i=1 \text { to } 7
\end{gathered}
$$

where $M_{i}$ is the expressions of the $i$ th moments of the normal distributions, see Table II.

\section{ACKNOWLEDGMENT}

The authors would like to thank the Power Factor Capacitor (PFC) Division Technical Department of CIRCUTOR S.A. for its contribution to this paper with the wind farm measurements.

\section{REFERENCES}

[1] S. A. Papathanassiou and M. P. Papadopoulos, "Harmonic analysis in a power system with wind generation," IEEE Trans. Power Del., vol. 21, no. 4, pp. 2006-2016, Oct. 2006.

[2] S. T. Tentzerakis and S. A. Papathanassiou, "An investigation of the har monic emissions of wind turbines," IEEE Trans. Energy Convers., vol. 22, no. 1, pp. 150-158, Mar. 2007.

[3] K.-H. Liu and L. Wang, "Analysis of measured harmonic currents and voltages contributed by a commercial wind power system," in Proc. IEEE Power Eng. Soc. Gen. Meeting, Tampa, FL, Jun. 2007, pp. 1-6.

[4] D. Schulz and R. E. Hanitsch, "Investigation of the current harmonic parameters of wind energy converters," presented at the IEEE Power Tech Conf., Bologna, Italy, Jun. 2003, vol. 3.

[5] T. Thiringer, T. Petru, and C. Liljegren, "Power quality impact of a sea located hybrid wind park," IEEE Trans. Energy Convers., vol. 16, no. 2, pp. 123-127, Jun. 2001.

[6] J. I. Herrera, T. W. Reddoch, and J. S. Lawler, "Harmonics generated by two variable speed wind generating systems," IEEE Trans. Energy Convers., vol. EC-3, no. 2, pp. 267-273, Jun. 1988.

[7] E. Muljadi, C. P. Butterfield, J. Chacon, and H. Romanowitz, "Power quality aspects in a wind power plant," in Proc. IEEE Power Eng. Soc. Gen. Meeting, Montreal, QC, Canada, Jun. 2006, p. 8.

[8] H. Emanuel, M. Schellschmidt, S. Wachtel, and S. Adloff, "Power quality measurements of wind energy converters with full-scale converter according to IEC 61400-21," in Proc. 10th Int. Conf. Elect. Power Quality Utilisation (EPQU), Lodz, Poland, Sep. 2009, pp. 1-7.

[9] Wind Turbine Generator Systems, Part 21: Measurement and Assessment of Power Quality Characteristics of Grid Connected Wind Turbines, 1st ed., IEC 61400-21, Dec. 2001.

[10] Electromagnetic Compatibility (EMC)_Part 4-7: Testing and Measurement Techniques-General Guide on Harmonics and Interharmonics Measurements and Instrumentation, for Power Supply Systems and Equipment Connected Thereto, 2nd ed., IEC 61000-4-7, Aug. 2002.

[11] Y. Baghzouz, R. F. Burch, A. Capasso, A. Cavallini, A. E. Emanuel M. Halpin, R. Langella, G. Montanari, K. J. Olejniczak, P. Ribeiro, S. Rios-Marcuello, F. Ruggiero, R. Thallam, A. Testa, and P. Verde, "Time-varying harmonics: Part II-Harmonic summation and propagation," IEEE Trans. Power Syst., vol. 17, no. 1, pp. 279-285, Jan. 2002.

[12] A. Cavallini, R. Langella, A. Testa, and F. Ruggiero, "Gaussian modeling of harmonic vectors in power systems," in Proc. 8th IEEE Int. Conf. Harmonics Quality Power (ICHQP), Athens, Greece, Oct. 1998, pp. 1010 1017.

[13] L. Pierrat and Y.-J. Wang, "Summation of randomly varying harmonicsTowards a univariate distribution function using generalized Gamma distribution," in Proc. 5th Int. Conf. Probabilistic Methods Appl. Power Syst. (PMAPS), Vancouver, BC, Canada, 1997, pp. 287-292.

[14] T. T. Soong, Fundamentals of Probability and Statistics for Engineers. Southern Gate, Chichester, U.K.: Wiley, 2004.

[15] E. W. Stacy, "A generalization of the Gamma distribution," Ann. Math. Stat., vol. 33, no. 3, pp. 1187-1192, Sep. 1962. 
[16] E. W. Stacy and G. A. Mihram, "Parameter estimation for a generalized Gamma distribution," Technometrics, vol. 7, no. 3, pp. 349-358, Aug. 1965.

[17] Electromagnetic Compatibility (EMC) - Part 3-6: Limits - Assessment of Emission Limits for the Connection of Distorting Installations to MV, HV and EHV Power Systems, IEC 61000-3-6, 2008 (Ed. 2).

[18] J. L. Blackburn, Symmetrical Components for Power Systems Engineering. New York: Marcel Dekker, 1993.

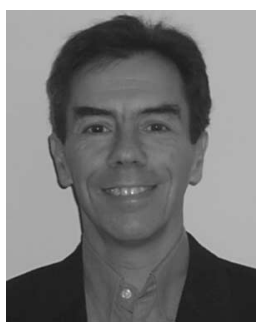

Luis Sainz was born in Barcelona (Spain) in 1965. He received the M.S. degree in industrial engineering and the Ph.D. degree in engineering from the Technical University of Catalonia (UPC), Barcelona, Spain, in 1990 and 1995, respectively.

Since 1991, he has been the Professor at the Department of Electrical Engineering, School of Industrial Engineering of Barcelona, UPC. His current research interests include power quality.

Prof. Sainz is the Coordinator of the Electrical Supply Quality Group at the UPC.

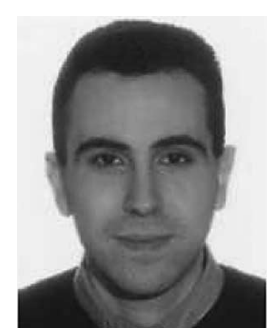

Juan Jose Mesas was born in Barcelona, Spain, in 1974. He received the B.S. degree in industrial engineering, the M.Res and the Ph.D. degrees in engineering from Technical University of Catalonia (UPC), Barcelona, Spain, in 2003, 2006, and 2010 respectively.

From 2004 to 2007, he worked as a Part-Time Associate Professor at the Departments of Applied Mathematics II and I of the UPC. Since 2007, he is currently working as a Research Fellow with a grant at the Department of Electrical Engineering, Escola Tècnica Superior d'Enginyeria Industrial de Barcelona, UPC. His current research interests include power system harmonics, numerical methods and optimization.

Mr. Mesas is a member of the Electrical Supply Quality (QSE) research group at the UPC.

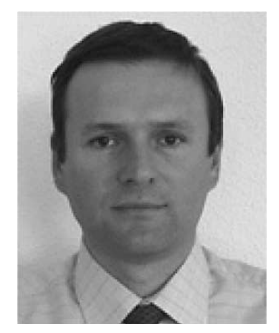

Remus Teodorescu (S'94-A'97-M'99-SM'02) received the Dipl. Ing. degree in electrical engineering from the Polytechnical University of Bucharest, Bucharest, Romania, in 1989, and the Ph.D. degree in power electronics from the University of Galati, Romania, in 1994.

In 1998, he joined the Institute of Energy Technology (Power Electronics Section), Aalborg University, Aalborg East, Denmark, where he is currently a Full Professor. He was the founder and is the Coordinator of the Green Power Laboratory, Aalborg University, where he is currently engaged in research on the development and testing of grid converters for renewable energy systems, and is the Coordinator of the Vestas Power Program. He is the author or coauthor of more than 120 papers published in various international journals and conference proceedings and one book. He is the holder of three patents (pending). His research interests include design and control of power converters used in renewable energy systems, distributed generation mainly wind power and photovoltaics, computer simulations, digital control implementation.

Prof. Teodorescu is an Associate Editor for the IEEE POWER ELECTRONICS LETTERS and the Chair of the IEEE Danish Joint Industrial Electronics Society/ Power Electronics Letters/Industry Applications Society (IAS) Chapter. He was the corecipient of the Technical Committee Prize Paper Awards at the IEEE IAS Annual Meeting 1998 and the Third-ABB Prize Paper Award at IEEE Optim 2002.

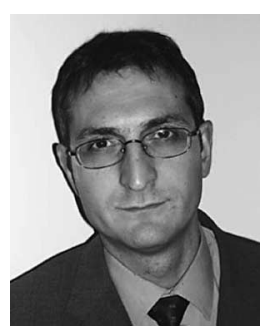

Pedro Rodriguez (S'99-M'04) received the B.S. degree in electrical engineering from the University of Granada, Granada, Spain, in 1989, and the M.S. and $\mathrm{Ph} . \mathrm{D}$. degrees in electrical engineering from the Technical University of Catalonia (UPC), Barcelona, Spain, in 1994 and 2004, respectively.

In 1990, he joined the faculty of UPC, as an Assistant Professor, where he is currently an Associate Professor at the Department of Electrical Engineering, Escola Universitària d'Enginyeria Tècnica Industrial de Terrassa. In 2005, he was a Visiting Researcher in the Center for Power Electronics Systems, Virginia Polytechnic Institute, Blacksburg. From 2006 and 2007, he was a Postdoctoral Researcher in the Institute of Energy Technology, Aalborg University (AAU), Aalborg East, Denmark, where he was a Lecturer of the Ph.D. courses in 2006. He is currently the Head of the Research Group on Renewable Electrical Energy Systems, UPC, and a Cosupervisor of the Vestas Power Programme in partnership collaboration with the AAU. He is the author or coauthor of more than 100 papers published in technical journals and conferences. He is the holder of four patents. His research interest include applying power electronics to distributed energy systems and power quality.

Dr. Rodriguez is a Member of the IEEE Power Electronics, IEEE Industry Application, IEEE Industrial Electronics Societies, and the IEEE Industrial Electronics Society Technical Committee on Renewable Energy Systems. He is an Associate Editor of the IEEE TRANSACTION ON POWER ELECTRONICS and the General Chair of the IEEE Industrial Electronics Student Forum. 